\title{
Multivariate Hardy and Littlewood inequalities on time scales
}

\author{
Ammara Nosheen and Aneela Nawaz \\ Department of Mathematics, University of Lahore (Sargodha Campus), \\ Sargodha, Pakistan, and \\ Khuram Ali Khan and Khalid Mahmood Awan \\ Department of Mathematics, University of Sargodha, Sargodha, Pakistan
}

Inequalities of multivariate Hardy and Littlewood

\begin{abstract}
In the paper we extend some Hardy and Littlewood type inequalities on time scales for the function of $n$ variables. Special cases of obtained results include generalized Wirtinger, Hardy and Littlewood type inequalities.
\end{abstract}

Keywords Hardy and Littlewood inequalities, Wirtinger type inequality, Time scales calculus

Paper type Orginal Article

\section{Introduction}

The discrete Hardy inequality [8] was proved and published by Hardy himself. It states that if $\left(c_{n}\right)$ is a sequence of non-negative real numbers which are not identically zero, then for every real number $p>1$, one has that

$$
\sum_{k=1}^{\infty}\left(\frac{c_{1}+c_{2}+c_{3}+\cdots+c_{k}}{k}\right)^{p}<\left(\frac{p}{p-1}\right)^{p} \sum_{k=1}^{\infty} c_{k}^{p} .
$$

The classical Hardy inequality [9] states that if $f \geq 0$ and integrable over any finite interval $(0, r)$ and $f^{d}$ is integrable and convergent over $(0, \infty)$ then for $d>1$,

$$
\int_{0}^{\infty}\left(\frac{1}{r} \int_{0}^{r} f(\tau) d \tau\right)^{d} d r \leq\left(\frac{d}{d-1}\right)^{d} \int_{0}^{\infty} f^{d}(r) d r
$$

JEL Classification — primary 26D15; secondary 39A13; 34N05

(c) Ammara Nosheen, Aneela Nawaz, Khuram Ali Khan and Khalid Mahmood Awan. Published in the Arab Journal of Mathematical Sciences. Published by Emerald Publishing Limited. This article is published under the Creative Commons Attribution (CC BY 4.0) license. Anyone may reproduce, distribute, translate and create derivative works of this article (for both commercial and non-commercial purposes), subject to full attribution to the original publication and authors. The full terms of this license may be seen at http://creativecommons.org/licences/by/4.0/legalcode

Declaration of Competing Interest: The authors declare that they have no known competing financial interests or personal relationships that could have appeared to influence the work reported in this paper.

Funding: This research did not receive any specific grant from funding agencies in the public, commercial, or not-for-profit sectors.

The publisher wishes to inform readers that the article "Multivariate Hardy and Littlewood inequalities on time scales" was originally published by the previous publisher of the Arab Journal of Mathematical Sciences and the pagination of this article has been subsequently changed. There has been no change to the content of the article. This change was necessary for the journal to transition from the previous publisher to the new one. The publisher sincerely apologises for any inconvenience caused. To access and cite this article, please use Nosheen, A., Nawaz, A., Khan, K. A., Awan, K. M. (2019), "Multivariate Hardy and Littlewood inequalities on time scales", Arab Journal of Mathematical Sciences, Vol. 26 No. 1/2, pp. 245-263. The original publication date for this paper was 27/12/2019.

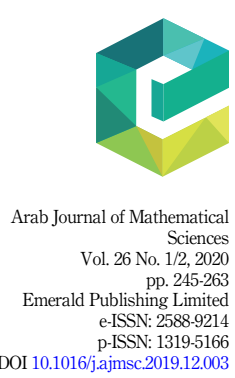


AJMS

$26,1 / 2$

equality holds if and only if $f(r)=0$ almost everywhere. Hardy inequality (1) has been generalized by Hardy himself in [11], where he exposed that, for any integrable function $f(y)>0$ on $(0, \infty)$ and $d>1$, the following hold

$$
\int_{0}^{\infty} \frac{1}{y^{n}}\left(\int_{y}^{\infty} f(h) d h\right)^{d} d y \leq\left(\frac{d}{1-n}\right)^{d} \int_{0}^{\infty} \frac{1}{y^{n-d}} f^{d}(y) d y, \quad n<1,
$$

$$
\int_{0}^{\infty} \frac{1}{y^{n}}\left(\int_{0}^{y} f(h) d h\right)^{d} d y \leq\left(\frac{d}{n-1}\right)^{d} \int_{0}^{\infty} \frac{1}{y^{n-d}} f^{d}(y) d y, \quad n>1 .
$$

Hardy and Littlewood [10] demonstrate the discrete versions of (2) and (3). In particular they proved that if $d>1$ and $\left(p_{m}\right)$ is a sequence of non-negative terms then

$$
\begin{aligned}
& \sum_{m=1}^{\infty} \frac{1}{m^{j}}\left(\sum_{i=m}^{\infty} p_{i}\right)^{d} \leq N \sum_{m=1}^{\infty} \frac{1}{m^{j-d}} p_{m}^{d}, \quad j<1, \\
& \sum_{m=1}^{\infty} \frac{1}{m^{j}}\left(\sum_{i=1}^{m} p_{i}\right)^{d} \leq N \sum_{m=1}^{\infty} \frac{1}{m^{j-d}} p_{m}^{d}, \quad j>1,
\end{aligned}
$$

where $N$ is a non-negative constant. Time scales calculus [12] was introduced in 1988 by the German mathematician Stefan Hilger, which unifies sums and integrals. Some extension of Hardy type inequalities on time scales can be found in [2-4].

S. H. Saker et al. [13] proved some Hardy and Littlewood type inequalities on time scales in the following form:

Theorem 1.1. Let $\mathbb{T}$ be a time scale with $a \in(0, \infty)_{\mathbb{T}}$ and $p, q>0$ such that $p / q \geq 2$ and $\gamma>1$. Furthermore assume that $g$ is a nonnegative and the delta integral $\int_{a}^{\infty} t^{\frac{p}{q}-\gamma} g^{p / q}(t) \Delta t$ exists. Let

$$
\Lambda(t)=\int_{a}^{t} g(s) \Delta s, \quad \text { for any } t \in[a, \infty]_{\mathbb{T}} .
$$

Then one gets

$$
\begin{aligned}
\int_{a}^{\infty} \frac{1}{t^{\gamma}}\left(\Lambda^{\sigma}(t)\right)^{p / q} \Delta t \leq & \frac{2^{\frac{p}{q}-2} p k^{\gamma}}{q(\gamma-1)}\left\{\int_{a}^{\infty} \frac{1}{t^{\gamma-\frac{p}{q}}} g^{p / q}(t) \Delta t\right\}^{\frac{p}{q}} \\
& \times\left\{\int_{a}^{\infty} \frac{\Lambda^{\sigma}(t) \Lambda^{p / q}}{t^{\gamma}} \Delta t\right\}^{\frac{p-q}{p}} \\
& +\frac{2^{\frac{p}{q}-2} p k^{\gamma}}{q(\gamma-1)} \int_{a}^{\infty} \frac{\mu^{\frac{p}{q}}-1}{t^{\gamma-1}} g^{p / q}(t) \Delta t .
\end{aligned}
$$

Theorem 1.2. Let $\mathbb{T}$ be a time scale with $a \in(0, \infty)_{\mathbb{T}}$ and $p, q>0$ such that $p / q \geq 2$ and $\gamma>1$. Furthermore assume that $g$ is a nonnegative function and the delta integral $\int_{a}^{\infty} t^{\frac{p}{q}-\gamma} g^{p / q}(t) \Delta t$ exist. Let $\Lambda(t)$ be as defined in (4). Then 


$$
\int_{a}^{\infty} \frac{1}{t^{\gamma}}\left(\Lambda^{\sigma}(t)\right)^{p / q} \Delta t \leq\left(\frac{2^{\frac{p}{q}-1} p k^{\gamma}}{q(\gamma-1)}\right)^{p / q} \int_{a}^{\infty} \frac{1}{t^{\gamma-\frac{p}{q}}} g^{p / q}(t) \Delta t .
$$

Inequalities of multivariate Hardy and Littlewood

Theorem 1.3. Let $\mathbb{T}$ be a time scale with $a \in(0, \infty)$ and $p, q>0$ such that $p / q>1$ and $\gamma>1$. Furthermore assume that $g$ is a nonnegative function and the delta integral $\int_{a}^{\infty} t^{\frac{p}{q}-\gamma} g^{p / q}(t) \Delta t$ exists. Let $\Lambda(t)$ be as defined in (4). Then

$$
\int_{a}^{\infty} \frac{1}{t^{\gamma}}\left(\Lambda^{\sigma}(t)\right)^{p / q} \Delta t \leq\left(\frac{p k^{\gamma}}{q(\gamma-1)}\right)^{p / q} \int_{a}^{\infty} \frac{1}{t^{\gamma-\frac{p}{q}}} g^{p / q}(t) \Delta t .
$$

Theorem 1.4. Let $\mathbb{T}$ be a time scale with $a \in(0, \infty)_{\mathbb{T}}$ and $p, q>0$ such that $p / q>1$ and $\gamma<1$. Furthermore assume that $g$ is a nonnegative and delta integral $\int_{a}^{\infty}(\sigma(t))^{\frac{p}{q}-\gamma} g^{p / q}(t) \Delta t$ exists. Let

$$
\Omega(t)=\int_{t}^{\infty} g(s) \Delta s, \quad \text { for any } t \in[a, \infty]_{\mathbb{T}} .
$$

Then one gets

$$
\int_{a}^{\infty} \frac{(\Omega(t))^{p / q}}{\sigma^{\gamma}(t)} \leq\left(\frac{p}{q(1-\gamma)}\right)^{p / q} \int_{a}^{\infty} \frac{g^{p / q}(t)}{(\sigma(t))^{\gamma-\frac{p}{q}}} \Delta t .
$$

In this paper we extend results of Theorem 1.1 to Theorem 1.4 for the function of $n$ variables.

\section{Preliminaries}

In this section, we recall the following concepts from theory of time scales [5,7]. A time scale is an arbitrary, non empty closed subset of real numbers. Set of integers and Cantor set are examples of time scales, while rational numbers, complex numbers and open interval between 0 and 1 not time scales. Let $\mathbb{T}$ be a time scale, for $t \in \mathbb{T}$, forward and backward jump operators are defined by

$$
\sigma(t):=\inf \{a \in \mathbb{T} ; \quad a>t\}, \quad \rho(t):=\sup \{a \in \mathbb{T} ; \quad a<t\},
$$

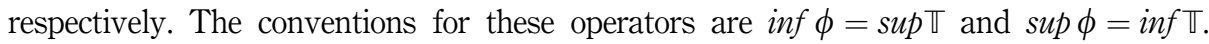
If $\sigma(t)>t$, then $t$ is right-scattered and if $\rho(t)<t$, then $t$ is left-scattered. Points that are rightscattered and left-scattered at the same time are called isolated points.

If $\sigma(t)=t$, then $t$ is right-dense and if $\rho(t)=t$, then $t$ is left-dense. Points that are rightdense and left-dense at the same time are called dense points. The functions $\mu: \mathbb{T} \rightarrow \mathbb{R}, \nu: \mathbb{T} \rightarrow \mathbb{R}$ defined by $\mu(t)=\sigma(t)-t$ and $\nu(t)=t-\rho(t)$ are called forward and backward graininess functions, respectively.

A function $g: \mathbb{T} \rightarrow \mathbb{R}$ is said to be right-dense continuous (rd-continuous) provided $g$ is continuous at right-dense points and at left-dense points in $\mathbb{T}$, left-hand limits exist and are finite. The set of all such rd-continuous functions is denoted by $C_{r d}(\mathbb{T})$. For any function $g: \mathbb{T} \rightarrow \mathbb{R}$, the notation $g^{\sigma}(t)$ denotes $g(\sigma(t))$. The delta derivative (also Hilger derivative) $g^{\Delta}(t)$ exists if and only if for every $\epsilon>0$ there exists a neighborhood $U$ of $t$ such that

$$
\left|g(\sigma(t))-g(s)-g^{\Delta}(t)(\sigma(t)-s)\right| \leq|\sigma(t)-s|, \quad \text { for alls, } \mathrm{t} \text { in } \mathrm{U} .
$$


AJMS

$26,1 / 2$

\section{8}

Assume that $h: \mathbb{T} \rightarrow \mathbb{R}$, if $H^{\Delta}(t)=h(t)$, then the Cauchy (delta) integral of $h$. defined by

$$
\int_{a}^{t} h(s) \Delta s:=H(t)-H(a) .
$$

Integration by parts formula [7, Theorem1.77]:

If $a, b \in \mathbb{T}$ and $u, v \in C_{r d}(\mathbb{T})$, then

$$
\int_{a}^{b} u(t) v^{\Delta}(t) \Delta t=[u(t) v(t)]_{a}^{b}-\int_{a}^{b} u^{\Delta}(t) v^{\sigma}(t) \Delta t .
$$

Chain rule 1 [7, Theorem 1.90$]$ :

Assume that $f: \mathbb{R} \rightarrow \mathbb{R}$ is continuously differentiable and suppose $g: \mathbb{T} \rightarrow \mathbb{R}$ is delta differentiable. Then $f$ og $: \mathbb{T} \rightarrow \mathbb{R}$ is delta differentiable and

$$
(f \circ g)^{\Delta}(t)=\left\{\int_{0}^{1} f^{\prime}\left(g(t)+h \mu(t) g^{\Delta}(t)\right) d h\right\} g^{\Delta}(t)
$$

holds.

Chain rule 2 [7, Theorem 1.87]:

If $f$ and $g$ satisfy the conditions of Chain rule 1 , Then $f \circ g: \mathbb{T} \rightarrow \mathbb{R}$ is delta differentiable and there exists $c$ in the real interval $[t, \sigma(t)]$ such that

$$
(f \circ g)^{\Delta}(t)=f^{\prime}(g(c)) g^{\Delta}(t) .
$$

Hölder's inequality [7, Theorem 6.13]:

For continuous real-valued functions $g: \mathbb{T} \rightarrow \mathbb{R}, h: \mathbb{T} \rightarrow \mathbb{R}$, let $a, b \in \mathbb{T}, p>1$ and $\frac{1}{p}+\frac{1}{q}=1$, then

$$
\int_{a}^{b} g(t) h(t) d t=\left(\int_{a}^{b} g^{p}(t) d t\right)^{1 / p}\left(\int_{a}^{b} h^{q}(t) d t\right)^{1 / q} .
$$

Fubini's Theorem on time scales [6]:

Let $\left(\psi, M, \mu_{\Delta}\right)$ and $\left(\Gamma, N, \lambda_{\Delta}\right)$ be two finite dimensional time scales measure spaces. If $\Lambda: \psi \times \Gamma \rightarrow \mathbb{R}$ is a $\mu_{\Delta} \times \lambda_{\Delta}$-integrable function. The function $\varsigma\left(t_{2}\right)=\int_{\psi} \Lambda\left(t_{1}, t_{2}\right) \Delta t_{1}$ exists for any $t_{1} \in \Gamma$ and $\xi\left(t_{1}\right)=\int_{\Gamma} \Lambda\left(t_{1}, t_{2}\right) \Delta t_{2}$ exists for $t_{2} \in \psi$, then

$$
\int_{\psi} \Delta t_{1} \int_{\Gamma} \Lambda\left(t_{1}, t_{2}\right) \Delta t_{2}=\int_{\Gamma} \Delta t_{2} \int_{\psi} \Lambda\left(t_{1}, t_{2}\right) \Delta t_{1} \text {. }
$$

We assume throughout that all the functions are non-negative and the integrals considered exist. with

In this paper, we use the following notations. We assume that there exists constant $k_{i}>0$

$$
\begin{gathered}
\frac{s_{i}}{\sigma_{i}\left(s_{i}\right)} \geq \frac{1}{k_{i}} \text { for } s_{i} \geq a_{i}, \quad i \in\{1, \ldots, n\} . \\
\Lambda_{k}^{\sigma_{1} \cdots \sigma_{j}}\left(t_{1}, \ldots, t_{n}\right) \doteq \Lambda_{k}^{\sigma_{1} \cdots \sigma_{j}} \doteq \Lambda_{k}\left(\sigma_{1}\left(t_{1}\right), \ldots, \sigma_{j}\left(t_{j}\right), t_{j+1}, \ldots, t_{n}\right), k, j \in\{1, \ldots, n\} \\
\int_{a_{1}}^{\infty} \ldots \int_{a_{n}}^{\infty} f\left(t_{1}, \ldots, t_{n}\right) \Delta t_{1}, \ldots, \Delta t_{n} \doteq \int_{\prod_{i=1}^{n} a_{i}}^{\infty} f\left(t_{1}, \ldots, t_{n}\right) \prod_{i=1}^{n} \Delta t_{i} .
\end{gathered}
$$


3. Hardy and Littlewood-type inequalities for $p / q \geq 2$ and $\gamma>1$

The following inequalities are used to prove next results.

$$
\begin{gathered}
a^{\lambda}+b^{\lambda} \leq(a+b)^{\lambda} \leq 2^{\lambda-1}\left(a^{\lambda}+b^{\lambda}\right) \text { for } a, b \geq 0, \lambda \geq 1 . \\
2^{\lambda-1}\left(a^{\lambda}+b^{\lambda}\right) \leq(a+b)^{\lambda} \leq a^{\lambda}+b^{\lambda} \text { for } a, b \geq 0,0 \leq \lambda \leq 1 .
\end{gathered}
$$

Inequalities of multivariate Hardy and Littlewood

Theorem 3.1. Assume $i \in\{1, \ldots, n\}, \mathbb{T}_{i}$ is a time scale with $a_{i} \in(0, \infty)_{\mathbb{T}_{i}}$ and $\gamma_{i}>1$, further assume $g:\left[a_{1}, \infty\right)_{\mathbb{T}_{1}} \times \cdots \times\left[a_{n}, \infty\right)_{\mathbb{T}_{n}} \rightarrow \mathbb{R}_{+}$is such that the delta integrals

$\int_{\prod_{i=1}^{n} a_{i}}^{\infty} \prod_{i=1}^{n}\left(t_{i}\right)^{\frac{p}{q}-\gamma_{i}} g^{p / q}\left(t_{1}, \ldots, t_{n}\right) \Delta t_{i}$ for any $\left(t_{1}, \ldots, t_{n}\right) \in\left[a_{1}, \infty\right)_{\mathbb{T}_{1}} \times \cdots \times\left[a_{n}, \infty\right)_{\mathbb{T}_{n}}$ exist, define

$$
\Lambda_{k}\left(t_{1}, \ldots, t_{n}\right)=\int_{\prod_{j=1}^{k} a_{j}}^{\infty} g\left(s_{1}, \ldots, s_{n}\right) \prod_{j=1}^{k} \Delta s_{j}, k \in\{1, \ldots, n\}
$$

then for $p, q>0$ and $p / q \geq 2$

$$
\begin{gathered}
\int_{i=1}^{\infty} \prod_{i}^{n} \frac{\left(\Lambda_{n}^{\sigma_{1} \ldots \sigma_{n}}\right)^{p / q}}{\prod_{i=1}^{n} t_{i}^{\gamma_{i}}} \prod_{i=1}^{n} \Delta t_{i} \\
\leq \sum_{r=1}^{n} \prod_{j=r+1}^{n} c_{j} \tilde{c}_{r} \int_{\prod_{j=r+1}^{n} a_{j}}^{\infty} \prod_{j=r+1}^{n} \frac{\left(\mu_{j}\left(t_{j}\right)\right)^{(p / q-1)}}{t_{j}^{\gamma_{j}-1}} \int_{\prod_{i=1}^{r-1} a_{i}}^{\infty} \prod_{i=1}^{r-1} \frac{1}{t_{i}^{\gamma_{i}}} \times \\
\\
\left\{\int_{a_{r}}^{\infty} \frac{\left(\Lambda_{r-1}^{\sigma_{1} \cdots \sigma_{r-1}}\right)^{p / q}}{t_{r}^{\gamma_{r}-p / q}} \Delta t_{r}\right\}^{q / p}\left\{\left(\Lambda_{r}^{\sigma_{1} \ldots \sigma_{r}}\right)\left(\Lambda_{r}^{\sigma_{1} \ldots \sigma_{r-1}}\right)^{p / q} \Delta t_{r}\right\}^{\frac{p-q}{p}} \prod_{i=1}^{r-1} \Delta t_{i} \prod_{j=r+1}^{n} \Delta t_{j} \\
+\prod_{i=1}^{n} \tilde{c}_{i} \int_{\prod_{i=1}^{n} a_{i}}^{\infty} \prod_{i=1}^{n} \frac{\left(\mu_{i}\left(t_{i}\right)\right)^{(p / q-1)}}{t_{i}^{\gamma_{i}-1}} g^{p / q}\left(t_{1}, \ldots, t_{n}\right) \prod_{i=1}^{n} \Delta t_{i}
\end{gathered}
$$

holds, where $\tilde{c}_{r}=c_{r} p / q, c_{r}=\frac{2^{p / q-2} k_{r}^{\gamma_{r}}}{\gamma_{r-1}}$.

Proof. To prove the result, we use the principle of mathematical induction. For $n=1$ the statement is true by Theorem 1.1. Let the statement be true for $1 \leq n \leq k$.

To prove the result for $n=k+1$. The left-hand side of (14) can be written as,

$$
\int_{i=1}^{\infty+1} a_{i} \frac{1}{\prod_{i=1}^{k+1} t_{i}^{\gamma_{i}}}\left(\Lambda_{k+1}^{\sigma_{1} \cdots \sigma_{k+1}}\right)^{p / q} \prod_{i=1}^{k+1} \Delta t_{i}
$$

Denote $\int_{a_{k+1}}^{\infty} \frac{\left(\Lambda_{k+1}^{\sigma_{1} \cdots \sigma_{k+1}}\right)^{p / q}}{t_{k+1}^{\gamma / k+1}} \Delta t_{k+1}=I_{k+1}$. Apply (5) with $\frac{\partial}{\Delta t_{k+1}} u\left(t_{k+1}\right)=\frac{1}{t_{k+1}^{\gamma k+1}}$ and

$$
\begin{gathered}
v^{\sigma_{k+1}}\left(t_{k+1}\right)=\left(\Lambda_{k+1}^{\sigma_{1} \cdots \sigma_{k+1}}\right)^{p / q} \text { by keeping fix }\left(t_{1}, \ldots, t_{k}\right) \in\left[a_{1}, \infty\right)_{\mathbb{T}_{1}} \times \cdots \times\left[a_{k}, \infty\right)_{\mathbb{T}_{k}} . \\
I_{k+1}=\left.\left[u\left(t_{k+1}\right)\left(\left(\Lambda_{k+1}^{\sigma_{1} \cdots \sigma_{k}}\right)^{p / q}\right)\right]\right|_{a_{k+1}} ^{\infty} \int_{a_{k+1}}^{\infty}-u\left(t_{k+1}\right) \frac{\partial}{\Delta t_{k+1}}\left(\Lambda_{k+1}^{\sigma_{1} \cdots \sigma_{k}}\right)^{p / q} \Delta t_{k+1},
\end{gathered}
$$


AJMS $\quad$ where, $\quad u\left(t_{k+1}\right)=\int_{t_{k+1}}^{\infty}-\frac{1}{s_{k+1}^{k_{k+1}}} \Delta s_{k+1}$.

Use chain rule (6) and the fact that $\sigma_{k+1}\left(s_{k+1}\right) \geq s_{k+1}$ to get

$$
\begin{aligned}
\frac{\partial}{\Delta s_{k+1}}\left(-\frac{1}{s_{k+1}^{\gamma_{k+1}-1}}\right)= & \left(\gamma_{k+1}-1\right) \int_{0}^{1}\left[h_{k+1} \sigma_{k+1}\left(s_{k+1}\right)+\left(1-h_{k+1}\right) s_{k+1}\right]^{-\gamma_{k+1}} d h_{k+1} \\
& \geq \frac{\left(\gamma_{k+1}-1\right)}{\sigma_{k+1}^{\gamma_{k+1}}\left(s_{k+1}\right)} .
\end{aligned}
$$

(10) together with (18) gives

$$
\frac{\partial}{\Delta s_{k+1}}\left(-\frac{1}{s_{k+1}^{\gamma k+1}-1}\right) \geq \frac{\left(\gamma_{k+1}-1\right)}{k_{k+1}^{\gamma_{k+1}} s_{k+1}^{\gamma / 1}} .
$$

Therefore

$$
\begin{aligned}
& \int_{t_{k+1}}^{\infty}-\frac{1}{s_{k+1}^{\gamma_{k+1}}} \Delta s_{k+1} \\
& \quad \geq \int_{t_{k+1}}^{\infty}-\frac{k_{k+1}^{\gamma_{k+1}}}{\gamma_{k+1}-1} \frac{\partial}{\Delta s_{k+1}}\left(-\frac{1}{s_{k+1}^{\gamma_{k+1}-1}}\right) \Delta s_{k+1}=-\frac{k_{k+1}^{\gamma_{k+1}}}{\gamma_{k+1}-1}\left(\frac{1}{t_{k+1}^{\gamma_{k+1}-1}}\right) .
\end{aligned}
$$

(17) together with (19) gives

$$
-u\left(t_{k+1}\right)=-\int_{t_{k+1}}^{\infty}-\frac{1}{s_{k+1}^{\gamma_{k+1}}} \Delta s_{k+1} \leq \frac{k_{k+1}^{\gamma_{k+1}}}{\gamma_{k+1}-1}\left(\frac{1}{t_{k+1}^{\gamma_{k+1}-1}}\right) .
$$

From (13), (16), (17), (20), we have (note that $u_{k+1}(\infty)=0$ and $\Lambda_{k+1}\left(t_{1}, \ldots, t_{k}, a_{k+1}\right)=0$ )

$$
I_{k+1}=\frac{k_{k+1}^{\gamma_{k+1}}}{\gamma_{k+1}-1} \int_{a_{k+1}}^{\infty} \frac{1}{t_{k+1}^{\gamma_{k+1}-1}} \frac{\partial}{\Delta t_{k+1}}\left(\Lambda_{k+1}^{\sigma_{1} \cdots \sigma_{k}}\right)^{p / q} \Delta t_{k+1} .
$$

Apply chain rule 1 (6) on the right-hand side of (21)

$$
\begin{aligned}
& \frac{\partial}{\Delta t_{k+1}}\left(\Lambda_{k+1}^{\sigma_{1} \cdots \sigma_{k}}\right)^{p / q} \\
& \quad=\frac{p}{q} \frac{\partial}{\Delta t_{k+1}} \Lambda_{k+1}^{\sigma_{1} \cdots \sigma_{k}} \int_{0}^{1}\left[\Lambda_{k+1}+h_{k+1} \mu_{k+1}\left(t_{k+1}\right) \frac{\partial}{\Delta t_{k+1}} \Lambda_{k+1}^{\sigma_{1} \cdots \sigma_{k}}\right]^{\frac{p}{q}-1} d h_{k+1} .
\end{aligned}
$$

Use right part of (11) on the right-hand side of (22),

$$
\begin{aligned}
& \frac{\partial}{\Delta t_{k+1}}\left(\Lambda_{k+1}^{\sigma_{1} \cdots \sigma_{k}}\right)^{p / q} \\
& \leq \frac{p}{q} 2^{p / q-2}\left(\Lambda_{k+1}^{\sigma_{1} \cdots \sigma_{k}}\right)^{p / q-1} \frac{\partial}{\Delta t_{k+1}}\left(\Lambda_{k+1}^{\sigma_{1} \cdots \sigma_{k}}\right) \\
& \quad+\frac{p}{q} 2^{p / q-2}\left(\mu_{k+1}\left(t_{k+1}\right)\right)^{p / q-1}\left(\frac{\partial}{\Delta t_{k+1}} \Lambda_{k+1}^{\sigma_{1} \cdots \sigma_{k}}\right) .
\end{aligned}
$$


Substitute (23) into (21)

$$
\begin{aligned}
& I_{k+1} \leq \frac{p 2^{p / q-2} k_{k+1}^{\gamma_{k+1}}}{q\left(\gamma_{k+1}-1\right)} \int_{a_{k+1}}^{\infty} \frac{1}{t_{k+1}^{\gamma_{k+1}-1}}\left(\Lambda_{k+1}^{\sigma_{1} \cdots \sigma_{k}}\right)^{p / q-1} \frac{\partial}{\Delta t_{k+1}} \Lambda_{k+1}^{\sigma_{1} \cdots \sigma_{k}} \Delta t_{k+1} \\
& +\frac{p 2^{p / q-2} k_{k+1}^{\gamma_{k+1}}}{q\left(\gamma_{k+1}-1\right)} \int_{a_{k+1}}^{\infty} \frac{1}{t_{k+1}^{\gamma_{k+1}-1}}\left(\mu_{k+1}\left(t_{k+1}\right)\right)^{p / q-1}\left(\frac{\partial}{\Delta t_{k+1}} \Lambda_{k+1}^{\sigma_{1} \cdots \sigma_{k}}\right)^{p / q} \Delta t_{k+1} .
\end{aligned}
$$

Since

$$
\frac{\partial}{\Delta t_{k+1}} \Lambda_{k+1}^{\sigma_{1} \cdots \sigma_{k}}=\Lambda_{k}^{\sigma_{1} \cdots \sigma_{k}} \geq 0
$$

Use (25) in (24)

$$
\begin{aligned}
I_{k+1} \leq & \frac{p 2^{p / q-2} k_{k+1}^{\gamma_{k+1}}}{q\left(\gamma_{k+1}-1\right)} \int_{a_{k+1}}^{\infty} \frac{1}{t_{k+1}^{\gamma_{k+1}-1}}\left(\Lambda_{k+1}^{\sigma_{1} \cdots \sigma_{k}}\right)^{p / q-1} \Lambda_{k}^{\sigma_{1} \cdots \sigma_{k}} \Delta t_{k+1} \\
& +\frac{p 2^{p / q-2} k_{k+1}^{\gamma_{k+1}}}{q\left(\gamma_{k+1}-1\right)} \int_{a_{k+1}}^{\infty} \frac{1}{t_{k+1}^{\gamma_{k+1}-1}}\left(\mu_{k+1}\left(t_{k+1}\right)\right)^{p / q-1}\left(\Lambda_{k}^{\sigma_{1} \cdots \sigma_{k}}\right)^{p / q} \Delta t_{k+1} .
\end{aligned}
$$

Substitute (26) in (15)

$$
\begin{aligned}
& \int_{\prod_{i=1}^{k+1} a_{i}}^{\infty} \frac{1}{\prod_{i=1}^{k+1} t_{i}^{\gamma_{i}}}\left(\Lambda_{k+1}^{\sigma_{1} \cdots \sigma_{k+1}}\right)^{p / q} \prod_{i=1}^{k+1} \Delta t_{i} \\
& \leq \int_{\prod_{i=1}^{k} a_{i}}^{\infty} \frac{1}{\prod_{i=1}^{k} t_{i}^{\gamma_{i}}} \frac{p 2^{p / q-2} k_{k+1}^{\gamma_{k+1}}}{q\left(\gamma_{k+1}-1\right)} \int_{a_{k+1}}^{\infty} \frac{1}{t_{k+1}^{\gamma_{k+1}-1}}\left(\Lambda_{k+1}^{\sigma_{1} \cdots \sigma_{k}}\right)^{p / q-1} \Lambda_{k}^{\sigma_{1} \cdots \sigma_{k}} \prod_{i=1}^{k} \Delta t_{k+1} \Delta t_{i} \\
& \quad+\int_{\prod_{i=1}^{k} a_{i}}^{\infty} \frac{1}{\prod_{i=1}^{k} t_{i}^{\gamma_{i}}} \frac{p 2^{p / q-2} k_{k+1}^{\gamma_{k+1}}}{q\left(\gamma_{k+1}-1\right)} \int_{a_{k+1}}^{\infty} \frac{\left(\mu_{k+1}\left(t_{k+1}\right)\right)^{p / q-1}}{t_{k+1}^{\gamma_{k+1}-1}}\left(\Lambda_{k}^{\sigma_{1} \cdots \sigma_{k}}\right)^{p / q} \prod_{i=1}^{k} \Delta t_{k+1} \Delta t_{i} .
\end{aligned}
$$

Exchange integrals on right-hand side of (27) $k$-times by using (9)

$$
\begin{gathered}
=\frac{p 2^{p / q-2} k_{k+1}^{\gamma_{k+1}}}{q\left(\gamma_{k+1}-1\right)} \int_{a_{k+1}}^{\infty} \frac{1}{t_{k+1}^{\gamma_{k+1}-1}} \int_{\prod_{i=1}^{k} a_{i}}^{\infty} \frac{1}{\prod_{i=1}^{k} t_{i}^{\gamma_{i}}}\left(\Lambda_{k+1}^{\sigma_{1} \cdots \sigma_{k}}\right)^{p / q-1} \Lambda_{k}^{\sigma_{1} \cdots \sigma_{k}} \prod_{i=1}^{k} \Delta t_{i} \Delta t_{k+1} \\
\quad+\frac{p 2^{p / q-2} k_{k+1}^{\gamma_{k+1}}}{q\left(\gamma_{k+1}-1\right)}\left(\mu_{k+1}\left(t_{k+1}\right)\right)^{p / q-1} \int_{a_{k+1}}^{\infty} \frac{1}{t_{k+1}^{\gamma_{k+1}-1}} \\
\quad \times \int_{\prod_{i=1}^{k} a_{i}}^{\infty} \frac{1}{\prod_{i=1}^{k} t_{i}^{\gamma_{i}}}\left(\Lambda_{k}^{\sigma_{1} \cdots \sigma_{k}}\right)^{p / q} \prod_{i=1}^{k} \Delta t_{i} \Delta t_{k+1} .
\end{gathered}
$$

Use the induction hypothesis with $\Lambda_{k}^{\sigma_{1} \cdots \sigma_{k}}$ in (28) for fixed $t_{k+1} \in \mathbb{T}_{k+1}$ and again apply (9) $k$-times to get 
AJMS

26,1/2

252

$$
\begin{aligned}
& =\frac{p 2^{p / q-2} k_{k+1}^{\gamma_{k+1}}}{q\left(\gamma_{k+1}-1\right)} \int_{a_{k+1}}^{\infty} \frac{1}{t_{k+1}^{\gamma_{k+1}-1}} \int_{\prod_{i=1}^{k} a_{i}}^{\infty} \frac{1}{\prod_{i=1}^{k} t_{i}^{\gamma_{i}}}\left(\Lambda_{k+1}^{\sigma_{1} \cdots \sigma_{k}}\right)^{p / q-1} \Lambda_{k}^{\sigma_{1} \cdots \sigma_{k}} \prod_{i=1}^{k} \Delta t_{i} \Delta t_{k+1} \\
& \quad+\frac{p 2^{p / q-2} k_{k+1}^{\gamma_{k+1}}}{q\left(\gamma_{k+1}-1\right)}\left(\mu_{k+1}\left(t_{k+1}\right)\right)^{p / q-1} \int_{a_{k+1}}^{\infty} \frac{1}{t_{k+1}^{\gamma_{k+1}-1}} \\
& \quad \times \sum_{r=1}^{k} \prod_{j=r+1}^{k} c_{j} \tilde{c}_{r} \int_{\prod_{j=r+1}^{k} a_{j}}^{\infty} \prod_{j=r+1}^{k} \frac{\left(\mu_{j}\left(t_{j}\right)\right)^{(p / q-1)}}{t_{j}^{\gamma_{j}-1}} \times \\
& \quad \prod_{i=1}^{\infty-1} a_{j} \prod_{i=1}^{r-1} \frac{1}{t_{i}^{\gamma_{i}}}\left\{\int_{a_{r}}^{\infty} \frac{\left(\Lambda_{r-1}^{\sigma_{1} \cdots \sigma_{r-1}}\right)^{p / q}}{t_{r}^{\gamma_{r}-p / q}} \Delta t_{r}\right\}^{q / p} \\
& \quad \times\left\{\left(\Lambda_{r}^{\sigma_{1} \cdots \sigma_{r}}\right)\left(\Lambda_{r}^{\sigma_{1} \cdots \sigma_{r-1}}\right)^{p / q} \Delta t_{r}\right\}^{\frac{p-q}{p}} \prod_{i=1}^{r-1} \Delta t_{i} \prod_{j=r+1}^{k} \Delta t_{j} \\
& \quad+\prod_{i=1}^{k} \tilde{c}_{i} \int_{\prod_{i=1}^{\infty} a_{j}}^{\infty} \prod_{i=1}^{k} \frac{\left(\mu_{i}\left(t_{i}\right)\right)^{(p / q-1)}}{t_{i}^{\gamma_{i}-1}} g^{p / q}\left(t_{1}, \ldots, t_{k}\right) \prod_{i=1}^{k} \Delta t_{i} .
\end{aligned}
$$

Hence

$$
\begin{aligned}
& \int_{\prod_{i=1}^{k+1} a_{i}}^{\infty} \frac{\left(\Lambda_{n}^{\sigma_{1} \ldots \sigma_{n}}\right)^{p / q}}{\prod_{i=1}^{k+1} t_{i}^{\gamma_{i}}} \prod_{i=1}^{k+1} \Delta t_{i} \\
& \leq \sum_{r=1}^{k+1} \prod_{j=r+1}^{k+1} c_{j} \tilde{c}_{r} \int_{\prod_{j=r+1}^{k+1} a_{j}}^{\infty} \prod_{j=r+1}^{k+1} \frac{\left(\mu_{j}\left(t_{j}\right)\right)^{(p / q-1)}}{t_{j}^{\gamma_{j}-1}} \int_{\prod_{i=1}^{r-1} a_{i}}^{\infty} \prod_{i=1}^{r-1} \frac{1}{t_{i}^{\gamma_{i}}} \times \\
& \left\{\int_{a_{r}}^{\infty} \frac{\left(\Lambda_{r-1}^{\sigma_{1} \cdots \sigma_{r-1}}\right)^{p / q}}{t_{r}^{\gamma_{r}-p / q}} \Delta t_{r}^{q / p}\left\{\Lambda_{r}^{\sigma_{1} \ldots \sigma_{r}}\left(\Lambda_{r}^{\sigma_{1} \ldots \sigma_{r-1}}\right)^{p / q} \Delta t_{r}\right\}^{\frac{p-q}{p}} \prod_{i=1}^{r-1} \Delta t_{i} \prod_{j=r+1}^{k+1} \Delta t_{j}\right. \\
& +\prod_{i=1}^{k+1} \tilde{c}_{i} \int_{\prod_{i=1}^{k+1} a_{i}}^{\infty} \prod_{i=1}^{k+1} \frac{\left(\mu_{i}\left(t_{i}\right)\right)^{(p / q-1)}}{t_{i}^{\gamma_{i}-1}} g^{p / q}\left(t_{1}, \ldots, t_{k+1}\right) \prod_{i=1}^{k+1} \Delta t_{i} .
\end{aligned}
$$

Hence by induction principle, the statement is true $\forall n \in \mathbb{N}$.

Theorem 3.2. Assume $i \in\{1, \ldots, n\}, \mathbb{T}_{i}$ is a time scale with $a_{i} \in(0, \infty)_{\mathbb{T}_{i}}$ and $\gamma_{i}>1$, further assume $g:\left[a_{1}, \infty\right)_{\mathbb{T}_{1}} \times \cdots \times\left[a_{n}, \infty\right)_{\mathbb{T}_{n}} \rightarrow \mathbb{R}_{+}$is such that the delta integrals $\int_{\prod_{i=1}^{n} a_{i}}^{n}$ $\prod_{i=1}^{n}\left(t_{i}\right)^{\frac{p}{q}-\gamma_{i}} g^{p / q}\left(t_{1}, \ldots, t_{n}\right) \prod_{i=1}^{n} \Delta t_{i}$ exist. Let $\Lambda_{k}\left(t_{1}, \ldots, t_{n}\right)$ be defined in (13), then for $p, q>0$ and $p / q \geq 2$

$$
\begin{aligned}
& \int_{\prod_{i=1}^{n} a_{i}}^{\infty} \frac{1}{\prod_{i=1}^{n} t_{i}^{\gamma_{i}}}\left(\Lambda_{n}^{\sigma_{1} \cdots \sigma_{n}}\right)^{p / q} \prod_{i=1}^{n} \Delta t_{i} \\
& \quad \leq\left(\frac{p}{q}\right)^{\frac{n p}{q}} \prod_{i=1}^{n}\left(\frac{2^{\frac{p}{q}-1} k_{i}^{\gamma_{i}}}{\left(\gamma_{i}-1\right)}\right)^{p / q} \int_{\prod_{i=1}^{n} a_{i}}^{\infty} \prod_{i=1}^{n} \frac{1}{t_{i}^{\gamma_{i}-p / q}} g^{p / q}\left(t_{1}, \ldots, t_{n}\right) \prod_{i=1}^{n} \Delta t_{i},
\end{aligned}
$$

holds. 
Proof. To prove the result, we use the principle of mathematical induction. For $n=1$ the statement is true by Theorem 1.2. Let the statement be true for $1 \leq n \leq k$.

To prove the result for $n=k+1$. Proceed it as in the proof of Theorem 3.1 up to (21). Apply chain rule 1 (6) on the right-hand side of (21) yields

$$
\begin{aligned}
& \frac{\partial}{\Delta t_{k+1}}\left(\Lambda_{k+1}^{\sigma_{1} \cdots \sigma_{k}}\right)^{p / q} \\
& \quad=\left(\frac{p}{q}\right) \frac{\partial}{\Delta t_{k+1}} \Lambda_{k+1}^{\sigma_{1} \cdots \sigma_{k}} \int_{0}^{1}\left[h_{k+1} \Lambda_{k+1}^{\sigma_{1} \cdots \sigma_{k+1}}+\left(1-h_{k+1}\right) \Lambda_{k+1}^{\sigma_{1} \cdots \sigma_{k}}\right]^{\frac{p}{q}-1} d h_{k+1} .
\end{aligned}
$$

Inequalities of multivariate Hardy and Littlewood

Use (11) on the right-hand side of (30),

$$
\leq\left(\frac{p}{q}\right) 2^{\frac{p}{q}-2}\left(\Lambda_{k+1}^{\sigma_{1} \cdots \sigma_{k+1}}\right)^{\frac{p}{q}-1} \frac{\partial}{\Delta t_{k+1}} \Lambda_{k+1}^{\sigma_{1} \cdots \sigma_{k}}+\left(\frac{p}{q}\right) 2^{\frac{p}{q}-2}\left(\Lambda_{k+1}^{\sigma_{1} \cdots \sigma_{k}}\right)^{\frac{p}{q}-1} \frac{\partial}{\Delta t_{k+1}} \Lambda_{k+1}^{\sigma_{1} \cdots \sigma_{k}},
$$

use the fact $\sigma_{k+1}\left(t_{k+1}\right) \geq t_{k+1}$

$$
\begin{aligned}
& =\left(\frac{p}{q}\right) 2^{\frac{p}{q}-2}\left(\Lambda_{k+1}^{\sigma_{1} \cdots \sigma_{k+1}}\right)^{\frac{p}{q}-1} \frac{\partial}{\Delta t_{k+1}} \Lambda_{k+1}^{\sigma_{1} \cdots \sigma_{k}}+\left(\frac{p}{q}\right) 2^{\frac{p}{q}-2}\left(\Lambda_{k+1}^{\sigma_{1} \cdots \sigma_{k+1}}\right)^{\frac{p}{q}-1} \frac{\partial}{\Delta t_{k+1}} \Lambda_{k+1}^{\sigma_{1} \cdots \sigma_{k}} \\
& =\left(\frac{p}{q}\right) 2^{\frac{p}{q}-1}\left(\Lambda_{k+1}^{\sigma_{1} \cdots \sigma_{k+1}}\right)^{\frac{p}{q}-1} \frac{\partial}{\Delta t_{k+1}} \Lambda_{k+1}^{\sigma_{1} \cdots \sigma_{k}} .
\end{aligned}
$$

Since

$$
\frac{\partial}{\Delta t_{k+1}} \Lambda_{k+1}^{\sigma_{1} \cdots \sigma_{k}}=\Lambda_{k}^{\sigma_{1} \cdots \sigma_{k}} \geq 0
$$

Use (32) in (31) and substitute in (21) to get

$$
I_{k+1} \leq \frac{p 2^{\frac{p}{q}-1} k_{k+1}^{\gamma_{k+1}}}{q\left(\gamma_{k+1}-1\right)} \int_{a_{k+1}}^{\infty} \frac{1}{t_{k+1}^{\gamma_{k+1}-1}}\left(\Lambda_{k+1}^{\sigma_{1} \cdots \sigma_{k+1}}\right)^{\frac{p}{q}-1} \Lambda_{k}^{\sigma_{1} \cdots \sigma_{k}} \Delta t_{k+1} .
$$

Apply Hölder's inequality on the right-hand side of (33) with indices $p / q$ and $p /(p-q)$

$$
I_{k+1} \leq \frac{p 2^{\frac{p}{q}-1} k_{k+1}^{\gamma_{k+1}}}{q\left(\gamma_{k+1}-1\right)}\left\{\int_{a_{k+1}}^{\infty}\left\{\frac{t_{k+1}^{\gamma_{k+1}\left(\frac{(p-q}{q}\right)}}{t_{k+1}^{\gamma_{k+1}-1}} \Lambda_{k}^{\sigma_{1} \cdots \sigma_{k}}\right\}^{p / q} \Delta t_{k+1}\right\}^{q / p} \times\left\{I_{k+1}\right\}^{\frac{p-q}{p}} .
$$

After simplification, we get

$$
I_{k+1} \leq\left(\frac{p 2^{\frac{p}{q}-1} k_{k+1}^{\gamma_{k+1}}}{q\left(\gamma_{k+1}-1\right)}\right)^{p / q} \int_{a_{k+1}}^{\infty} \frac{\left(\Lambda_{k}^{\sigma_{1} \cdots \sigma_{k}}\right)^{p / q}}{t_{k+1}^{-\frac{p}{q}+\gamma_{k+1}}} \Delta t_{k+1} .
$$


AJMS

$26,1 / 2$

254

Substitute (34) into (15)

$$
\begin{aligned}
& \int_{\prod_{i=1}^{k+1} a_{i}}^{\infty} \frac{1}{\prod_{i=1}^{k+1} t_{i}^{\gamma_{i}}}\left(\Lambda_{k+1}^{\sigma_{1} \cdots \sigma_{k+1}}\right)^{p / q} \prod_{i=1}^{k+1} \Delta t_{i} \\
& \quad \leq \int_{\prod_{i=1}^{k} a_{i}}^{\infty} \frac{1}{\prod_{i=1}^{k} t_{i}^{\gamma_{i}}}\left(\frac{p 2^{\frac{p}{q}-1} k_{k+1}^{\gamma_{k+1}}}{q\left(\gamma_{k+1}-1\right)}\right)^{p / q} \int_{a_{k+1}}^{\infty} \frac{\left(\Lambda_{k}^{\sigma_{1} \cdots \sigma_{k}}\right)^{p / q}}{t_{k+1}^{-\frac{p}{q}+\gamma_{k+1}}} \Delta t_{k+1} .
\end{aligned}
$$

Exchange integrals on right-hand side of (35) $k$-times by using (9)

$$
\left(\frac{p 2^{q^{-}-1} k_{k+1}^{\gamma_{k+1}}}{q\left(\gamma_{k+1}-1\right)}\right)^{p / q} \int_{a_{k+1}}^{\infty} \frac{1}{t_{k+1}^{-\frac{p}{q}+\gamma_{k+1}}}\left\{\int_{\prod_{i=1}^{k} a_{i}}^{\infty} \frac{1}{\prod_{i=1}^{k} t_{i}^{\gamma_{i}}}\left(\Lambda_{k}^{\sigma_{1} \cdots \sigma_{k}}\right)^{p / q} \prod_{i=1}^{k} \Delta t_{i}\right\} \Delta t_{k+1} .
$$

Use the induction hypothesis for $\Lambda_{k}^{\sigma_{1} \cdots \sigma_{k}}$ in (36) for fixed $t_{k+1} \in \mathbb{T}_{k+1}$ and again apply (9) $k$ times to get

$$
\begin{aligned}
& \int_{i=1}^{\infty} a_{i}^{k+1} \frac{1}{\prod_{i=1}^{k+1} t_{i}^{\gamma_{i}}}\left(\Lambda_{k+1}^{\sigma_{1} \cdots \sigma_{k+1}}\right)^{p / q} \prod_{i=1}^{k+1} \Delta t_{i} \\
& \quad \leq\left(\frac{p}{q}\right)^{\frac{(k+1) p}{q}} \prod_{i=1}^{k+1}\left(\frac{2^{\frac{p}{q}-1} k_{i}^{\gamma_{i}}}{\gamma_{i}-1}\right)^{p / q} \int_{\prod_{i=1}^{k+1} a_{i}}^{\infty} \prod_{i=1}^{k+1} \frac{1}{t_{i}^{\gamma_{i}-p / q}} g^{p / q}\left(t_{1}, \ldots, t_{k+1}\right) \prod_{i=1}^{k} \Delta t_{i} .
\end{aligned}
$$

Hence by induction principle, the statement is true $\forall n \in \mathbb{N}$.

Corollary 3.3. As a special case of Theorem 3.2, when $\mathbb{T}_{1}=\cdots=\mathbb{T}_{n}=\mathbb{R}, p / q=\lambda>1$ and $\gamma_{i}<1$, (29) becomes the following Wirtinger type inequality

$$
\begin{aligned}
\int_{i=1}^{\infty} a_{i} & \frac{1}{\prod_{i=1}^{n} t_{i}^{\gamma_{i}}}\left(G\left(t_{1}, \ldots, t_{n}\right)\right)^{\lambda} \prod_{i=1}^{n} d t_{i} \\
\quad \leq & \prod_{i=1}^{n}\left(\frac{\lambda 2^{\lambda-1}}{1-\gamma_{i}}\right)^{\lambda} \int_{\prod_{i=1}^{n} a_{i}}^{\infty} \frac{1}{\prod_{i=1}^{n} t_{1}^{\gamma_{i}-\lambda}}\left(\frac{\partial^{n}}{\partial t_{1} \cdots \partial t_{n}} G^{\lambda}\left(t_{1}, \ldots, t_{n}\right)\right) \prod_{i=1}^{n} d t_{i},
\end{aligned}
$$

where $G\left(t_{1}, \ldots, t_{n}\right) \doteq \int_{\prod_{i=1}^{n} a_{i}}^{t_{i}} g\left(s_{1}, \ldots, s_{n}\right) \prod_{i=1}^{n} d s_{i}$.

When $\gamma_{1}=\cdots=\gamma_{n}=\lambda>1$, we have another Hardy type inequality for function of n-variables

$$
\begin{gathered}
\int_{\prod_{i=1}^{n} a_{i}}^{\infty} \frac{1}{\prod_{i=1}^{n} t_{i}}\left(\int_{\prod_{i=1}^{n} a_{i}}^{t_{i}} g\left(s_{1}, \ldots, s_{n}\right) \prod_{i=1}^{n} d s_{i}\right)^{\lambda} \prod_{i=1}^{n} d t_{i} \\
\leq\left(\frac{\lambda 2^{\lambda-1}}{\lambda-1}\right)^{\lambda} g^{\lambda}\left(t_{1}, \ldots, t_{n}\right) \prod_{i=1}^{n} d t_{i} .
\end{gathered}
$$


Remark 3.4. Assume that $\mathbb{T}_{1}=\cdots=\mathbb{T}_{n}=\mathbb{N}$ in Theorem $3.2, p / q=\lambda>1, a_{i}>1$, $\gamma_{i}>1$ for $i \in\{1, \ldots, n\}$, further assume that $\sum_{m_{1}=1}^{\infty} \ldots \sum_{m_{n}=1}^{\infty} g^{\lambda}\left(m_{1}, \ldots, m_{n}\right)$ is convergent. (29) becomes the following discrete Hardy and Littlewood inequality

$$
\begin{aligned}
\sum_{m_{1}=1}^{\infty} & \cdots \sum_{m_{n}=1}^{\infty} \frac{1}{m_{1}^{\gamma_{1}} \ldots m_{n}^{\gamma_{n}}}\left(\sum_{k_{1}=1}^{m_{1}} \ldots \sum_{k_{n}=1}^{m_{n}} g\left(k_{1}, \ldots, k_{n}\right)\right)^{\lambda} \\
& \leq \prod_{i=1}^{n}\left(\frac{2^{\lambda-1} \lambda}{\gamma_{i}-1}\right)^{\lambda} \sum_{m_{1}=1}^{\infty} \cdots \sum_{m_{n}=1}^{\infty} \frac{1}{\prod_{i=1}^{n} m_{i}^{\gamma_{i}-\lambda}} g^{\lambda}\left(m_{1}, \ldots, m_{n}\right) .
\end{aligned}
$$

\section{Hardy and Littlewood-type inequalities for $p / q \geq 1$ and $\gamma>1$}

Theorem 4.1. Assume $i \in\{1, \ldots, n\}, \mathbb{T}_{i}$ is a time scale with $a_{i} \in(0, \infty)_{\mathbb{T}_{i}}$ and $\gamma_{i}<1$, further assume $g:\left[a_{1}, \infty\right)_{\mathbb{T}_{1}} \times \cdots \times\left[a_{n}, \infty\right)_{\mathbb{T}_{n}} \rightarrow \mathbb{R}_{+}$is such that the delta integrals $\int_{\prod_{i=1}^{n} a_{i}}^{n}$ $\prod_{i=1}^{n} t_{i}^{\frac{p}{q}-\gamma_{i}} g^{p / q}\left(t_{1}, \ldots, t_{n}\right) \prod_{i=1}^{n} \Delta t_{i}$ exist. Let $\Lambda_{k}\left(t_{1}, \ldots, t_{n}\right)$ be defined in (13), then for $p, q>0$ and $p / q>1$

$$
\begin{aligned}
& \int_{\prod_{i=1}^{n} a_{i}}^{\infty} \frac{1}{\prod_{i=1}^{n} t_{i}^{\gamma_{i}}}\left(\Lambda_{n}^{\sigma_{1} \cdots \sigma_{n}}\right)^{p / q} \prod_{i=1}^{n} \Delta t_{i} \\
& \quad \leq\left(\frac{p}{q}\right)^{\frac{n p}{q}} \prod_{i=1}^{n}\left(\frac{k_{i}^{\gamma_{i}}}{\gamma_{i}-1}\right)^{p / q} \int_{\prod_{i=1}^{n} a_{i}}^{\infty} \prod_{i=1}^{n} \frac{1}{t_{i}^{\gamma_{i}-p / q}} g^{p / q}\left(t_{1}, \ldots, t_{n}\right) \prod_{i=1}^{n} \Delta t_{i},
\end{aligned}
$$

holds, where $n$ is a positive integer.

Proof. To prove the result, we use the principle of mathematical induction. For $n=1$ the statement is true by Theorem 1.3. Let the statement be true for $1 \leq n \leq k$.

To prove the result for $n=k+1$. Proceed it as in the proof of Theorem 3.1 up to (21). Apply the chain rule $2(7)$ to get

$$
\frac{\partial}{\Delta t_{k+1}}\left(\Lambda_{k+1}^{\sigma_{1} \cdots \sigma_{k}}\right)^{p / q}=\frac{p}{q}\left(\Lambda_{k+1}^{\sigma_{1} \cdots \sigma_{k}}\left(t_{1}, \ldots, t_{k}, c_{k+1}\right)\right)^{\frac{p}{q}-1} \frac{\partial}{\Delta t_{k+1}} \Lambda_{k+1}^{\sigma_{1} \cdots \sigma_{k}},
$$

where $c_{k+1} \in\left[t_{k+1}, \sigma_{k+1}\left(t_{k+1}\right)\right]$. Since

$$
\frac{\partial}{\Delta t_{k+1}} \Lambda_{k+1}^{\sigma_{1} \cdots \sigma_{k}} \doteq \Lambda_{k}^{\sigma_{1} \cdots \sigma_{k}} \geq 0,
$$

and $\sigma_{k+1}\left(t_{k+1}\right) \geq c_{k+1}$, one has that

$$
\frac{\partial}{\Delta t_{k+1}} \Lambda_{k+1}^{\sigma_{1} \cdots \sigma_{k}} \leq \frac{p}{q}\left(\Lambda_{k+1}^{\sigma_{1} \cdots \sigma_{k+1}}\right)^{\frac{p}{q}-1} \Lambda_{k}^{\sigma_{1} \cdots \sigma_{k}} .
$$

Substitute (38) into (21)

$$
I_{k+1} \leq \frac{p k_{k+1}^{\gamma_{k+1}}}{q\left(\gamma_{k+1}-1\right)} \int_{a_{k+1}}^{\infty} \frac{\left(\Lambda_{k+1}^{\sigma_{1} \cdots \sigma_{k+1}}\right)^{\frac{p}{q}-1}}{t_{k+1}^{\gamma_{k+1}-1}} \Lambda_{k}^{\sigma_{1} \cdots \sigma_{k}} \Delta t_{k+1} .
$$


AJMS

$26,1 / 2$

Apply Hölder's inequality on the right-hand side of (39) with indices $p / q$ and $p /(p-q)$

$$
I_{k+1} \leq \frac{p k_{k+1}^{\gamma_{k+1}}}{q\left(\gamma_{k+1}-1\right)}\left\{\int_{a_{k+1}}^{\infty}\left\{\frac{t_{k+1}^{\gamma_{k+1}\left(\frac{p-q}{p}\right)}}{t_{k+1}^{\gamma_{k+1}-1}} \Lambda_{k}^{\sigma_{1}, \ldots, \sigma_{k}}\right\}^{p / q} \Delta t_{k+1}\right\}^{q / p} \times\left\{I_{k+1}\right\}^{\frac{p-q}{p}} .
$$

After simplification, we get

$$
I_{k+1} \leq\left(\frac{p k_{k+1}^{\gamma_{k+1}}}{q\left(\gamma_{k+1}-1\right)}\right)^{p / q} \int_{a_{k+1}}^{\infty} \frac{\left(\Lambda_{k}^{\sigma_{1}, \ldots, \sigma_{k}}\right)^{p / q}}{t_{k+1}^{-\frac{p}{q}+\gamma_{k+1}}} \Delta t_{k+1} .
$$

Substitute (40) into (15)

$$
\begin{aligned}
& \int_{\prod_{i=1}^{k+1} a_{i}}^{\infty} \frac{1}{\prod_{i=1}^{k+1} t_{i}^{\gamma_{i}}}\left(\Lambda_{k+1}^{\sigma_{1} \cdots \sigma_{k+1}}\right)^{p / q} \prod_{i=1}^{k+1} \Delta t_{i} \\
& \quad \leq \int_{\prod_{i=1}^{k} a_{i}}^{\infty} \frac{1}{\prod_{i=1}^{k} t_{i}^{\gamma_{i}}}\left(\frac{p k_{k+1}^{\gamma_{k+1}}}{q\left(\gamma_{k+1}-1\right)}\right)^{p / q} \int_{a_{k+1}}^{\infty} \frac{\left(\Lambda_{k}^{\sigma_{1}, \ldots, \sigma_{k}}\right)^{p / q}}{t_{k+1}^{\gamma_{k+1}-\frac{p}{q}}} \Delta t_{k+1} .
\end{aligned}
$$

Exchange integrals on right-hand side of (41) $k$-times by using (9)

$$
=\left(\frac{p k_{k+1}^{\gamma_{k+1}}}{q\left(\gamma_{k+1}-1\right)}\right)^{p / q} \int_{a_{k+1}}^{\infty} \frac{1}{t_{k+1}^{\gamma_{k+1}-\frac{p}{q}}}\left\{\int_{\prod_{i=1}^{k} a_{i}}^{\infty} \frac{1}{\prod_{i=1}^{k} t_{i}^{\gamma_{i}}}\left(\Lambda_{k}^{\sigma_{1} \cdots \sigma_{k}}\right)^{p / q} \prod_{i=1}^{k} \Delta t_{i}\right\} \Delta t_{k+1} .
$$

Use the induction hypothesis with $\left(\Lambda_{k}^{\sigma_{1} \cdots \sigma_{k}}\right)^{p / q}$ in (42) for fixed $t_{k+1} \in \mathbb{T}_{k+1}$ and again apply (9) $k$-times to get

$$
\begin{aligned}
& \int_{\prod_{i=1}^{k+1} a_{i}}^{\infty} \frac{1}{\prod_{i=1}^{k+1} t_{i}^{\gamma_{i}}}\left(\Lambda_{k+1}^{\sigma_{1} \cdots \sigma_{k+1}}\right)^{p / q} \prod_{i=1}^{k+1} \Delta t_{i} \\
& \quad \leq\left(\frac{p}{q}\right)^{\frac{(k+1) p}{q}} \prod_{i=1}^{k+1}\left(\frac{k_{i}^{\gamma_{i}}}{\gamma_{i}-1}\right)^{p / q} \int_{\prod_{i=1}^{k+1} a_{i}}^{\infty} \prod_{i=1}^{k+1} \frac{1}{t_{i}^{\gamma_{i}-p / q}} g^{p / q}\left(t_{1}, \ldots, t_{k+1}\right) \prod_{i=1}^{k+1} \Delta t_{i} .
\end{aligned}
$$

Hence by induction principle, the statement is true $\forall \quad n \in \mathbb{N}$.

Corollary 4.2. As a special case of Theorem 4.1 , when $\mathbb{T}_{1}=\cdots=\mathbb{T}_{n}=\mathbb{R}, p / q=\lambda>1$ and $\gamma_{1}, \ldots, \gamma_{n}<1$, (37) becomes the following Wirtinger type inequality,

$$
\begin{aligned}
& \int_{\prod_{i=1}^{n}}^{\infty} a_{i} \frac{1}{\prod_{i=1}^{n} t_{i}^{\gamma_{i}}} G^{\lambda}\left(t_{1}, \ldots, t_{n}\right) \prod_{i=1}^{n} d t_{i} \\
& \quad \leq \prod_{i=1}^{n}\left(\frac{\lambda}{1-\gamma_{i}}\right)^{\lambda} \int_{\prod_{i=1}^{n} a_{i}}^{\infty} \frac{1}{\prod_{i=1}^{n} t_{1}^{\gamma_{i}-\lambda}}\left(\frac{\partial^{n}}{\partial t_{1} \cdots \partial t_{n}} G^{\lambda}\left(t_{1}, \ldots, t_{n}\right)\right) \prod_{i=1}^{n} d t_{i},
\end{aligned}
$$

where $G\left(t_{1}, \ldots, t_{n}\right) \doteq \int_{\prod_{i=1}^{n} a_{i}}^{t_{i}} g\left(s_{1}, \ldots, s_{n}\right) \prod_{i=1}^{n} \Delta s_{i}$. 
When $\gamma_{1}=\cdots=\gamma_{n}=\lambda>1$, we have the classical Hardy type inequality for function of $n$-variables

$$
\begin{gathered}
\int_{\prod_{i=1}^{n} a_{i}}^{\infty} \frac{1}{\prod_{i=1}^{n} t_{i}}\left(\int_{\prod_{i=1}^{n} a_{i}}^{t_{i}} g\left(s_{1}, \ldots, s_{n}\right) \prod_{i=1}^{n} d s_{i}\right)^{\lambda} \prod_{i=1}^{n} d t_{i} \\
\leq\left(\frac{\lambda}{\lambda-1}\right)^{\lambda} g^{\lambda}\left(t_{1}, \ldots, t_{n}\right) \prod_{i=1}^{n} d t_{i} .
\end{gathered}
$$

Corollary 4.3. Assume that $\mathbb{T}_{1}=\cdots=\mathbb{T}_{n}=\mathbb{N}$ in Theorem $4.1, p / q=\lambda>1, a_{i}>1$, $\gamma_{i}>1$ for $i \in\{1, \ldots, n\}$, further assume that $\sum_{m_{1}=1}^{\infty} \cdots \sum_{m_{n}=1}^{\infty} g^{\lambda}\left(m_{1}, \ldots, m_{n}\right)$ is convergent. Note that in this case $\frac{m_{i}}{\sigma_{i}\left(m_{i}\right)}=\frac{m_{i}}{m_{i}+1}$ therefore $\frac{1}{2} \leq \frac{m_{i}}{m_{i}+1} \leq 1$, and we get following discrete Hardy and Littlewood inequality

$$
\begin{aligned}
& \sum_{m_{1}=1}^{\infty} \cdots \sum_{m_{n}=1}^{\infty} \frac{1}{m_{1}^{\gamma_{1}} \ldots m_{n}^{\gamma_{n}}}\left(\sum_{k_{1}=1}^{m_{1}} \ldots \sum_{k_{n}=1}^{m_{n}} g\left(k_{1}, \ldots, k_{n}\right)\right)^{\lambda} \\
& \quad \leq \prod_{i=1}^{n}\left(\frac{2^{\lambda} \lambda}{\gamma_{i}-1}\right)^{\lambda} \sum_{m_{1}=1}^{\infty} \cdots \sum_{m_{n}=1}^{\infty} \frac{1}{\prod_{i=1}^{n} m_{i}^{\gamma_{i}-\lambda}} g^{\lambda}\left(m_{1}, \ldots, m_{n}\right) .
\end{aligned}
$$

Remark 4.4. Assume $i \in\{1, \ldots, n\}, \mathbb{T}_{i}$ is a time scale with $a_{i} \in(0, \infty)_{\mathbb{T}_{i}}$ and $\gamma_{i}<1$, further assume $g:\left[a_{1}, \infty\right)_{\mathbb{T}_{1}} \times \cdots \times\left[a_{n}, \infty\right)_{\mathbb{T}_{n}} \rightarrow \mathbb{R}_{+}$is such that the delta integrals $\int_{\prod_{i=1}^{\infty} a_{i}}^{n} \prod_{i=1}^{n} \sigma_{i}\left(t_{i}\right)^{\frac{p}{q}-\gamma_{i}}\left(\frac{\sigma_{i}\left(t_{i}\right)}{t_{i}}\right)^{\frac{p}{q}\left(\gamma_{i}-1\right)} g_{n}^{p / q}\left(t_{1}, \ldots, t_{n}\right) \prod_{i=1}^{n} \Delta t_{i}$ exist. Let $\Lambda_{k}\left(t_{1}, \ldots, t_{n}\right)$ be defined in Theorem 3.1, then for $p, q>0$ and $p / q>1$

$$
\begin{aligned}
& \int_{\prod_{i=1}^{n} a_{i}}^{\infty} \frac{\left(\Lambda_{n}^{\sigma_{1} \cdots \sigma_{n}}\right)^{p / q}}{\prod_{i=1}^{n}\left(\sigma_{i}\left(t_{i}\right)\right)^{\gamma_{i}}} \prod_{i=1}^{n} \Delta t_{i} \\
& \quad \leq\left(\frac{p}{q}\right)^{\frac{n p}{q}} \prod_{i=1}^{n}\left(\frac{1}{\gamma_{i}-1}\right)^{p / q} \int_{\prod_{i=1}^{n} a_{i}}^{\infty} \frac{g^{p / q}\left(t_{1}, \ldots, t_{n}\right)}{\prod_{i=1}^{n} \sigma^{\gamma_{i}-\frac{p}{q}}\left(t_{i}\right)} \prod_{i=1}^{n}\left(\frac{\sigma_{i}\left(t_{i}\right)}{t_{i}}\right)^{\frac{p}{q}\left(\gamma_{i}-1\right)} \prod_{i=1}^{n} \Delta t_{i},
\end{aligned}
$$

holds.

Proof. Replace left-hand side of (37) in Theorem 4.1 by

$$
\prod_{\prod_{i=1}^{n} a_{i}}^{\infty} \frac{\left(\Lambda_{n}^{\sigma_{1} \cdots \sigma_{n}}\right)^{p / q}}{\prod_{i=1}^{n}\left(\sigma_{i}\left(t_{i}\right)\right)^{\gamma_{i}}} \prod_{i=1}^{n} \Delta t_{i},
$$

and proceed as in the proof of Theorem 4.1.

\section{Hardy and Littlewood-type inequalities for $p / q \leq 2$ and $\gamma>1$}

Theorem 5.1. Assume $i \in\{1, \ldots, n\}, \mathbb{T}_{i}$ is a time scale with $a_{i} \in(0, \infty)_{\mathbb{T}_{i}}$ and $\gamma_{i}<1$, further assume $g:\left[a_{1}, \infty\right)_{\mathbb{1}_{1}} \times \cdots \times\left[a_{n}, \infty\right)_{\mathbb{T}_{n}} \rightarrow \mathbb{R}_{+}$is such that the delta integrals 
AJMS

$26,1 / 2$

258

$\int_{\prod_{i=1}^{n} a_{i}}^{\infty} \prod_{i=1}^{n}\left(t_{i}\right)^{\frac{p}{q}-\gamma_{i}} g^{p / q}\left(t_{1}, \ldots, t_{n}\right) \prod_{i=1}^{n} \Delta t_{i}$ exist. Let $\Lambda_{k}\left(t_{1}, \ldots, t_{n}\right)$ be defined in (13), then for $p, q>0$ and $p / q \leq 2$

$$
\int_{\prod_{i=1}^{n} a_{i}}^{\infty} \frac{1}{\prod_{i=1}^{n} t_{i}^{\gamma_{i}}}\left(\Lambda_{n}^{\sigma_{1} \cdots \sigma_{n}}\right)^{p / q} \prod_{i=1}^{n} \Delta t_{i}
$$

$$
\leq\left(\frac{p}{q}\right)^{\frac{n p}{q}} \prod_{i=1}^{n}\left(\frac{2 k_{i}^{\gamma_{i}}}{\left(\gamma_{i}-1\right)}\right)^{p / q} \int_{\prod_{i=1}^{n} a_{i}}^{\infty} \frac{1}{\prod_{i=1}^{n} t_{i}^{\gamma_{i}-\frac{g}{q}}} g^{p / q}\left(t_{1}, \ldots, t_{n}\right) \prod_{i=1}^{n} \Delta t_{i} .
$$

Proof. Proceed as in the proof of Theorem 3.2 and apply inequality (12) in (21) to get (43).

Remark 5.2. As a special case of Theorem 5.1, when $\mathbb{T}_{1}=\cdots=\mathbb{T}_{n}=\mathbb{R}, p / q=\lambda>1$ and $\gamma_{1}, \ldots, \gamma_{n}<1$, we have the following Hardy type inequality

$$
\begin{gathered}
\int_{\prod_{i=1}^{n} a_{i}}^{\infty} \frac{1}{\prod_{i=1}^{n} t_{i}^{\gamma_{i}}}\left(\int_{\prod_{i=1}^{t_{i}} a_{i}}^{t_{i}} g\left(s_{1}, \ldots, s_{n}\right) \prod_{i=1}^{n} d s_{i}\right)^{\lambda} \prod_{i=1}^{n} d t_{i} \\
\leq \prod_{i=1}^{n}\left(\frac{2 \lambda}{1-\gamma_{i}}\right)^{\lambda} \int_{\prod_{i=1}^{n} a_{i}}^{\infty} \frac{1}{\prod_{i=1}^{n} t_{i}^{\gamma_{i}-\lambda}} g^{\lambda}\left(t_{1}, \ldots, t_{n}\right) \prod_{i=1}^{n} d t_{i} .
\end{gathered}
$$

Remark 5.3. Assume that $\mathbb{T}_{1}=\cdots=\mathbb{T}_{n}=\mathbb{N}$ in Theorem 5.1, $p / q=\lambda>1, a_{i}>1$, $\gamma_{i}>1$ for $i \in\{1, \ldots, n\}$, further assume that $\sum_{m_{1}=1}^{\infty} \cdots \sum_{m_{n}=1}^{\infty} g^{\lambda}\left(m_{1}, \ldots, m_{n}\right)$ is convergent. In this case, (43) becomes the following discrete Hardy and Littlewood inequality

$$
\begin{aligned}
& \sum_{m_{1}=1}^{\infty} \ldots \sum_{m_{n}=1}^{\infty} \frac{1}{m_{1}^{\gamma_{1}} \ldots m_{n}^{\gamma_{n}}}\left(\sum_{k_{1}=1}^{m_{1}} \ldots \sum_{k_{n}=1}^{m_{n}} g\left(k_{1}, \ldots, k_{n}\right)\right)^{\lambda} \\
& \quad \leq \prod_{i=1}^{n}\left(\frac{2 \lambda}{\gamma_{i}-1}\right)^{\lambda} \sum_{m_{1}=1}^{\infty} \cdots \sum_{m_{n}=1}^{\infty} \frac{1}{\prod_{i=1}^{n} m_{i}^{\gamma_{i}-\lambda}} g^{\lambda}\left(m_{1}, \ldots, m_{n}\right) .
\end{aligned}
$$

\section{Hardy and Littlewood-type inequalities for $p / q>1$ and $\gamma<1$}

Theorem 6.1. Assume $i \in\{1, \ldots, n\}, \mathbb{T}_{i}$ is a time scale with $a_{i} \in(0, \infty)$ and $\gamma_{i}<1$, further assume $g:\left[a_{1}, \infty\right)_{\mathbb{T}_{1}} \times \cdots \times\left[a_{n}, \infty\right)_{\mathbb{T}_{n}} \rightarrow \mathbb{R}_{+}$is such that the delta integrals $\int_{\prod_{i=1}^{n} a_{i}}^{\infty} \prod_{i=1}^{n}\left(\sigma_{i}\left(t_{i}\right)\right)^{\frac{b}{-}-\gamma_{i}} g^{p / q}\left(t_{1}, \ldots, t_{n}\right) \prod_{i=1}^{n} \Delta t_{i}$ exist, for any $\left(t_{1}, \ldots, t_{n}\right) \in\left[a_{1}, \infty\right)_{\mathbb{T}_{1}} \times \cdots \times$ $\left[a_{n}, \infty\right)_{\mathbb{W}_{n}}$, define

$$
\Omega_{k}\left(t_{1}, \ldots, t_{n}\right)=\int_{\prod_{j=1}^{k} a_{j}}^{t_{j}} g\left(s_{1}, \ldots, s_{n}\right) \prod_{j=1}^{k} \Delta s_{j}, \quad k \in\{1, \ldots, n\}
$$

then for $p, q>0$ and $p / q>1$ 


$$
\begin{aligned}
& \int_{\prod_{i=1}^{n} a_{i}}^{\infty} \frac{\Omega_{n}^{p / q}\left(t_{1}, \ldots, t_{n}\right)}{\prod_{i=1}^{n} \sigma_{i}^{\gamma_{i}}\left(t_{i}\right)} \prod_{i=1}^{n} \Delta t_{i} \\
& \quad \leq\left(\frac{p}{q}\right)^{\frac{n p}{q}} \prod_{i=1}^{n}\left(\frac{1}{1-\gamma_{i}}\right)^{p / q} \int_{\prod_{i=1}^{n} a_{i}}^{\infty} \frac{1}{\prod_{i=1}^{n}\left(\sigma_{i}\left(t_{i}\right)\right)^{\gamma_{i}-p / q}} g^{p / q}\left(t_{1}, \ldots, t_{n}\right) \prod_{i=1}^{n} \Delta t_{i}
\end{aligned}
$$

Inequalities of multivariate Hardy and Littlewood

holds, where $n$ is any positive integer.

Proof. To prove the result, we use the principle of mathematical induction. For $n=1$ the statement is true by Theorem 1.4. Let the statement be true for $1 \leq n \leq k$.

To prove the result for $n=k+1$. The left-hand side of (45) can be written as

$$
\int_{\prod_{i=1}^{n} a_{i}}^{\infty} \frac{\Omega_{k+1}^{p / q}\left(t_{1}, \ldots, t_{k+1}\right)}{\prod_{i=1}^{k+1} \sigma_{i}^{\gamma_{i}}\left(t_{i}\right)} \prod_{i=1}^{k+1} \Delta t_{i}
$$

Denote $\int_{a_{k+1}}^{\infty} \frac{\Omega_{k+1}^{p / q}\left(t_{1}, \ldots, t_{k+1}\right)}{\sigma_{k+1}^{\gamma k+1}\left(t_{k+1}\right)} \Delta t_{k+1}=I_{k+1}$. Apply (5) with $\frac{\partial}{\Delta t_{k+1}} v\left(t_{k+1}\right)=\frac{1}{\sigma_{k+1}^{\gamma_{k+1}\left(t_{k+1}\right)}}$ and $u\left(t_{k+1}\right)=$ $\Omega_{k+1}^{p / q}\left(t_{1}, \ldots, t_{k+1}\right)$. Thus

$$
\begin{aligned}
I_{k+1} & =\left.v\left(t_{k+1}\right) \Omega_{k+1}^{p / q}\left(t_{1}, \ldots, t_{k+1}\right)\right|_{a_{k+1}} ^{\infty} \\
& +\int_{a_{k+1}}^{\infty} v^{\sigma_{k+1}}\left(t_{k+1}\right)\left(-\frac{\partial}{\Delta t_{k+1}} \Omega_{k+1}^{p / q}\left(t_{1}, \ldots, t_{k+1}\right)\right) \Delta t_{k+1},
\end{aligned}
$$

where $v\left(t_{k+1}\right)=\int_{a_{k+1}}^{t_{k+1}} 1 / \sigma_{k+1}^{\gamma_{k+1}}\left(s_{k+1}\right) \Delta s_{k+1}$. Use chain rule (6) and the fact that $\sigma_{k+1}\left(s_{k+1}\right) \geq s_{k+1}$ to get

$$
\begin{aligned}
\frac{\partial}{\Delta s_{k+1}}\left(s_{k+1}^{1-\gamma_{k+1}}\right) & =\left(1-\gamma_{k+1}\right) \int_{0}^{1}\left[h_{k+1} \sigma_{k+1}\left(s_{k+1}\right)+\left(1-h_{k+1}\right) s_{k+1}\right]^{-\gamma_{k+1}} d h_{k+1} \\
& \geq\left(1-\gamma_{k+1}\right) \frac{1}{\sigma_{k+1}^{\gamma k+1}\left(s_{k+1}\right)},
\end{aligned}
$$

which gives

$$
v^{\sigma_{k+1}}\left(t_{k+1}\right)=\int_{a_{k+1}}^{\sigma_{k+1}\left(t_{k+1}\right)} \frac{1}{\sigma_{k+1}^{\gamma_{k+1}}\left(s_{k+1}\right)} \Delta s_{k+1} \leq \frac{1}{\left(1-\gamma_{k+1}\right)}\left(\sigma_{k+1}\left(t_{k+1}\right)\right)^{1-\gamma_{k+1}} .
$$

Combine (47), (48) and use the facts $\Omega_{k+1}\left(t_{1}, \ldots, t_{k}, \infty\right)=0, v\left(a_{k+1}\right)=0$ to get

$$
I_{k+1} \leq \frac{1}{\left(1-\gamma_{k+1}\right)} \int_{a_{k+1}}^{\infty} \frac{-\frac{\partial}{\Delta t_{k+1}} \Omega_{k+1}^{p / q}\left(t_{1}, \ldots, t_{k+1}\right)}{\left(\sigma_{k+1}\left(t_{k+1}\right)\right)^{\gamma_{k+1}-1}} \Delta t_{k+1} .
$$

Apply chain rule 2 (7) to find

$$
-\frac{\partial}{\Delta t_{k+1}} \Omega_{k+1}^{p / q}\left(t_{1}, \ldots, t_{k+1}\right)=-\left(\frac{p}{q}\right) \Omega_{k+1}^{\frac{p}{q}-1}\left(t_{1}, \ldots, t_{k}, c_{k+1}\right) \frac{\partial}{\Delta t_{k+1}} \Omega_{k+1}\left(t_{1}, \ldots, t_{k+1}\right),
$$

where, $c_{k+1} \in\left[t_{k+1}, \sigma_{k+1}\left(t_{k+1}\right)\right]$. Since 
AJMS

26,1/2

$$
\begin{aligned}
\frac{\partial}{\Delta t_{k+1}} \Omega_{k+1}\left(t_{1}, \ldots, t_{k+1}\right) & =-\int_{\prod_{i=1}^{k} a_{i}}^{\infty} g\left(s_{1}, \ldots, s_{k}, t_{k+1}\right) \prod_{i=1}^{k} \Delta s_{i} \\
& \doteq \Omega_{k}\left(t_{1}, \ldots, t_{k+1}\right) \leq 0,
\end{aligned}
$$

and $c_{k+1} \geq t_{k+1}$, one has that

$$
-\frac{\partial}{\Delta t_{k+1}} \Omega_{k+1}^{p / q}\left(t_{1}, \ldots, t_{k+1}\right) \leq \frac{p}{q} \Omega_{k+1}^{\frac{p}{q}-1}\left(t_{1}, \ldots, t_{k+1}\right) \Omega_{k}\left(t_{1}, \ldots, t_{k+1}\right) .
$$

Substitute (50) into (49)

$$
I_{k+1} \leq \frac{p}{q\left(1-\gamma_{k+1}\right)} \int_{a_{k+1}}^{\infty} \frac{\Omega_{k+1}^{\frac{p}{q}-1}\left(t_{1}, \ldots, t_{k+1}\right)}{\left(\sigma_{k+1}\left(t_{k+1}\right)\right)^{\gamma_{k+1}-1}} \Omega_{k}\left(t_{1}, \ldots, t_{k+1}\right) \Delta t_{k+1} .
$$

Apply Hölder's inequality on the right-hand side of (51) with indices $p / q$ and $p /(p-q)$ to obtain

$$
\begin{aligned}
& I_{k+1} \leq \frac{p}{q\left(1-\gamma_{k+1}\right)}\left[\int_{a_{k+1}}^{\infty}\left[\frac{\left(\sigma_{k+1}^{\gamma_{k+1}}\left(t_{k+1}\right)\right)^{\frac{p-q}{p}}}{\left(\sigma_{k+1}\left(t_{k+1}\right)\right)^{\gamma_{k+1}-1}} \Omega_{k}\left(t_{1}, \ldots, t_{k+1}\right)\right]^{p / q} \Delta t_{k+1}\right]^{q / p} \\
& \quad \times\left[I_{k+1}\right]^{\frac{p-q}{p}} .
\end{aligned}
$$

After simplification, we get

$$
I_{k+1} \leq\left(\frac{p}{q\left(1-\gamma_{k+1}\right)}\right)^{p / q} \int_{a_{k+1}}^{\infty} \frac{\Omega_{k}^{p / q}\left(t_{1}, \ldots, t_{k+1}\right)}{\left(\sigma_{k+1}\left(t_{k+1}\right)\right)^{-\frac{p}{q}+\gamma_{k+1}}} \Delta t_{k+1} .
$$

Substitute (52) into (46)

$$
\begin{aligned}
& \int_{i=1}^{\infty} a_{i}^{k+1} \frac{\Omega_{k+1}^{p / q}\left(t_{1}, \ldots, t_{k+1}\right)}{\prod_{i=1}^{k+1} \sigma_{i}^{\gamma_{i}}\left(t_{i}\right)} \prod_{i=1}^{k+1} \Delta t_{i} \\
& \quad \leq \int_{\prod_{i=1}^{k} a_{i}}^{\infty} \frac{1}{\prod_{i=1}^{k} \sigma_{i}^{\gamma_{i}}\left(t_{i}\right)}\left(\frac{p}{q\left(1-\gamma_{k+1}\right)}\right)^{p / q} \int_{a_{k+1}}^{\infty} \frac{\Omega_{k}^{p / q}\left(t_{1}, \ldots, t_{k+1}\right)}{\left(\sigma_{k+1}\left(t_{k+1}\right)\right)^{-\frac{p}{q}+\gamma_{k+1}}} \prod_{i=1}^{k+1} \Delta t_{i} .
\end{aligned}
$$

Exchange integrals on right-hand side of (53) $k$-times by using (9)

$$
\begin{aligned}
= & \left(\frac{p}{q\left(1-\gamma_{k+1}\right)}\right)^{p / q} \int_{a_{k+1}}^{\infty} \frac{1}{\left(\sigma_{k+1}\left(t_{k+1}\right)\right)^{-\frac{p}{q}+\gamma_{k+1}}} \\
& \times\left\{\int_{\prod_{i=1}^{k} a_{i}}^{\infty} \frac{\Omega_{k}^{p / q}\left(t_{1}, \ldots, t_{k+1}\right)}{\prod_{i=1}^{k} \sigma_{i}^{\gamma_{i}}\left(t_{i}\right)} \prod_{i=1}^{k} \Delta t_{i}\right\} \Delta t_{k+1} .
\end{aligned}
$$


Use the induction hypothesis for $\Omega_{k}\left(t_{1}, \ldots, t_{k+1}\right)$ in (54) instead for $\Omega_{k}\left(t_{1}, \ldots, t_{k}\right)$ for fixed $t_{k+1} \in \mathbb{T}_{k+1}$ and again apply (9) $k$ times to get

$$
\begin{aligned}
& \int_{\prod_{i=1}^{k+1} a_{i}}^{\infty} \frac{\Omega_{k+1}^{p / q}\left(t_{1}, \ldots, t_{k+1}\right)}{\prod_{i=1}^{k+1} \sigma_{i}^{\gamma_{i}}\left(t_{i}\right)} \prod_{i=1}^{k+1} \Delta t_{i} \\
& \quad \leq\left(\frac{p}{q}\right)^{\frac{(k+1) p}{q}} \prod_{i=1}^{k+1}\left(\frac{1}{1-\gamma_{i}}\right)^{p / q} \int_{\prod_{i=1}^{k+1} a_{i}}^{\infty} \prod_{i=1}^{k+1} \frac{1}{\left(\sigma_{i}\left(t_{i}\right)\right)^{\gamma_{i}-p / q}} g^{p / q}\left(t_{1}, \ldots, t_{k+1}\right) \prod_{i=1}^{k+1} \Delta t_{i} .
\end{aligned}
$$

Hence by induction principle, the statement is true $\forall n \in \mathbb{N}$.

Corollary 6.2. Under the conditions of Theorem 6.1, we get the following inequality

$$
\begin{aligned}
& \int_{i=1}^{\infty} a_{i} \frac{1}{\prod_{i=1}^{n} \sigma_{i}^{\gamma_{i}}\left(t_{i}\right)}\left(\Omega_{n}\left(\sigma_{1}\left(t_{1}\right), \ldots, \sigma_{n}\left(t_{n}\right)\right)\right)^{p / q} \prod_{i=1}^{n} \Delta t_{i} \\
& \leq\left(\frac{p}{q}\right)^{\frac{n p}{q}} \prod_{i=1}^{n}\left(\frac{1}{1-\gamma_{i}}\right)^{p / q} \int_{\prod_{i=1}^{n} a_{i}}^{\infty} \prod_{i=1}^{n} \frac{1}{\left(\sigma_{i}\left(t_{i}\right)\right)^{\gamma_{i}-p / q}} g^{p / q}\left(t_{1}, \ldots, t_{n}\right) \prod_{i=1}^{n} \Delta t_{i} .
\end{aligned}
$$

Proof. The fact $\frac{\partial^{n} \Omega_{n}}{\Delta t_{1} \cdots \Delta t_{n}} \leq 0$ implies

$$
\begin{gathered}
\int_{\prod_{i=1}^{n} a_{i}}^{\infty} \frac{1}{\prod_{i=1}^{n} \sigma_{i}^{\gamma_{i}}\left(t_{i}\right)}\left(\Omega_{n}\left(\sigma_{1}\left(t_{1}\right), \ldots, \sigma_{n}\left(t_{n}\right)\right)\right)^{p / q} \prod_{i=1}^{n} \Delta t_{i} \\
\leq \int_{\prod_{i=1}^{n} a_{i}}^{\infty} \frac{1}{\prod_{i=1}^{n} \sigma_{i}^{\gamma_{i}}\left(t_{i}\right)}\left(\Omega_{n}\left(t_{1}, \ldots, t_{n}\right)\right)^{p / q} \prod_{i=1}^{n} \Delta t_{i} .
\end{gathered}
$$

Now use (45) in (56) to get (55).

Remark 6.3. Consider $\mathbb{T}_{1}=\cdots=\mathbb{T}_{n}=\mathbb{R}, p / q=\lambda>1$ and $\gamma_{1}, \ldots, \gamma_{n}<1$, in Theorem 6.1. Denote $G\left(t_{1}, \ldots, t_{n}\right)=\int_{\prod_{i=1}^{\infty} t_{i}}^{n} g\left(s_{1}, \ldots, s_{n}\right) \prod_{i=1}^{n} d s_{i}$. Thus, (45) takes the form

$$
\begin{aligned}
& \int_{\prod_{i=1}^{n} a_{i}}^{\infty} \frac{1}{\prod_{i=1}^{n} t_{i}^{\gamma_{i}}}\left(G^{\lambda}\left(t_{1}, \ldots, t_{n}\right)\right) \prod_{i=1}^{n} d t_{i} \\
& \quad \leq \prod_{i=1}^{n}\left(\frac{\lambda}{1-\gamma_{i}}\right)^{\lambda} \int_{\prod_{i=1}^{n} a_{i}}^{\infty} \frac{1}{\prod_{i=1}^{n}\left(t_{i}\right)^{\gamma_{i}-\lambda}} \frac{\partial^{n}}{\partial t_{1} \ldots \partial t_{n}} G^{\lambda}\left(t_{1}, \ldots, t_{n}\right) \prod_{i=1}^{n} d t_{i},
\end{aligned}
$$

which can be considered as a generalization of Wirtinger's inequality [1].

Remark 6.4. As a special case of Theorem 6.1 , assume that $\mathbb{T}_{1}=\cdots=\mathbb{T}_{n}=\mathbb{N}$, $p / q=\lambda>1, a_{1}=\cdots=a_{n}=1$ and $\gamma_{1}, \ldots, \gamma_{n}<1$. In this case (55) becomes the following discrete Hardy and Littlewood inequality 
AJMS

$26,1 / 2$

262

$$
\begin{aligned}
& \sum_{m_{1}=1}^{\infty} \cdots \sum_{m_{n}=1}^{\infty} \frac{1}{\prod_{i=1}^{n}\left(m_{i}+1\right)^{\gamma_{i}}}\left(\sum_{k_{1}=m_{1}+1}^{\infty} \ldots \sum_{k_{n}=m_{n}+1}^{\infty} g\left(k_{1}, \ldots, k_{n}\right)\right)^{\lambda} \\
& \quad \leq \prod_{i=1}^{n}\left(\frac{\lambda}{1-\gamma_{i}}\right)^{\lambda} \sum_{m_{1}=1}^{\infty} \cdots \sum_{m_{n}=1}^{\infty} \frac{1}{\prod_{i=1}^{n}\left(m_{i}+1\right)^{\gamma_{i}-\lambda}} g^{\lambda}\left(m_{1}, \ldots, m_{n}\right) .
\end{aligned}
$$

\section{Hardy and Littlewood-type inequalities for $p / q \leq 2$ and $\gamma<1$}

Theorem 7.1. Assume $i \in\{1, \ldots, n\}, \mathbb{T}_{i}$ is a time scale with $a_{i} \in(0, \infty)_{\mathbb{T}_{i}}$ and $\gamma_{i}<1$, further assume $g:\left[a_{1}, \infty\right)_{\mathbb{T}_{1}} \times \cdots \times\left[a_{n}, \infty\right)_{\mathbb{T}_{n}} \rightarrow \mathbb{R}_{+}$is such that the delta integrals $\int_{\prod_{i=1}^{n} a_{i}}^{\infty} \prod_{i=1}^{n}\left(\sigma_{i}\left(t_{i}\right)\right)^{\frac{\underline{p}}{q}-\gamma_{i}} g^{p / q}\left(t_{1}, \ldots, t_{n}\right) \prod_{i=1}^{n} \Delta t_{i}$ exist, then for $p, q>0$ and $p / q \leq 2$ Then

$$
\begin{aligned}
& \int_{\prod_{i=1}^{n} a_{i}}^{\infty} \frac{1}{\prod_{i=1}^{n} \sigma_{i}^{\gamma_{i}}\left(t_{i}\right)}\left(\int_{\prod_{i=1}^{n} t_{i}}^{\infty} g\left(s_{1}, \ldots, s_{n}\right) \prod_{i=1}^{n} \Delta s_{i}\right)^{p / q} \prod_{i=1}^{n} \Delta t_{i} \\
& \leq\left(\frac{p}{q}\right)^{\frac{n p}{q}} \prod_{i=1}^{n}\left(\frac{2}{1-\gamma_{i}}\right)^{p / q} \int_{\prod_{i=1}^{n} a_{i}}^{\infty} \prod_{i=1}^{n} \frac{1}{\left(\sigma_{i}\left(t_{i}\right)\right)^{\gamma_{i}-p / q}} g^{p / q}\left(t_{1}, \ldots, t_{n}\right) \prod_{i=1}^{n} \Delta t_{i} .
\end{aligned}
$$

Proof: Use (12) and proceed as in the proof of Theorem 6.1 to get (57).

Remark 7.2. In Theorem 7.1, when $\mathbb{T}_{1}=\cdots=\mathbb{T}_{n}=\mathbb{R}, p / q=\lambda>1$ and $\gamma_{i}<1$, (57) becomes the following Wirtinger type inequality,

$$
\begin{aligned}
& \int_{\prod_{i=1}^{n} a_{i}}^{\infty} \frac{1}{\prod_{i=1}^{n} t_{i}^{\gamma_{i}}}\left(G\left(t_{1}, \ldots, t_{n}\right)\right)^{\lambda} \prod_{i=1}^{n} d t_{i} \\
& \quad \leq \prod_{i=1}^{n}\left(\frac{2 \lambda}{1-\gamma_{i}}\right)^{\lambda} \int_{\prod_{i=1}^{n} a_{i}}^{\infty} \frac{1}{\prod_{i=1}^{n}\left(t_{i}\right)^{\gamma_{i}-\lambda}}\left(\frac{\partial^{n}}{\partial t_{1} \ldots \partial t_{n}} G\left(t_{1}, \ldots, t_{n}\right)\right)^{\lambda} \prod_{i=1}^{n} d t_{i},
\end{aligned}
$$

where $G\left(t_{1}, \ldots, t_{n}\right) \doteq \int_{\prod_{i=1}^{n} t_{i}}^{\infty} g\left(s_{1}, \ldots, s_{n}\right) \prod_{i=1}^{n} d s_{i}$.

Remark 7.3. In Theorem 7.1, assume that $\mathbb{T}_{1}=\cdots=\mathbb{T}_{n}=\mathbb{N}, p / q=\lambda>1$, $a_{1}=\cdots=a_{n}=1$ and $\gamma_{i}<1$. (57) becomes the following discrete Hardy and Littlewood inequality

$$
\begin{aligned}
& \sum_{m_{1}=1}^{\infty} \cdots \sum_{m_{n}=1}^{\infty} \frac{1}{\prod_{i=1}^{n}\left(m_{i}+1\right)^{\gamma_{i}}}\left(\sum_{k_{1}=m_{1}+1}^{\infty} \cdots \sum_{k_{n}=m_{n}+1}^{\infty} g\left(k_{1}, \ldots, k_{n}\right)\right)^{\lambda} \\
& \quad \leq \prod_{i=1}^{n}\left(\frac{2 \lambda}{1-\gamma_{i}}\right)^{\lambda} \sum_{m_{1}=1}^{\infty} \cdots \sum_{m_{n}=1}^{\infty} \frac{1}{\prod_{i=1}^{n}\left(m_{i}+1\right)^{\gamma_{i}-\lambda}} g^{\lambda}\left(m_{1}, \ldots, m_{n}\right) .
\end{aligned}
$$

\section{References}

[1] R.P. Agarwal, M. Bohner, D. O'Regan, S.H. Saker, Some Wirtinger-type inequalities on time scales and their applications, Pacific J. Math. 252 (2011) 1-26. 
[2] W. Ahmad, K.A. Khan, A. Nosheen, M.A. Sultan, Copson, Leindler type inequalities of function of several variables on time scales, Punjab Univ. J. Math. 51 (8) (2019) 157-168.

[3] M.S. Ashraf, K.A. Khan, A. Nosheen, Hardy-Copson type inequalities on time scales for the functions of $n$ independent variables, Int. J. Anal. Appl. 17 (2) (2019) 244-259, http://dx.doi.org/10. 28924/2291-8639-17-2019-244.

[4] J. Baric, R. Bibi, M. Bohner, A. Nosheen, J. Pecaric, Jensen inequalities and their applications on time scales, in: Element, Zagreb, Croatia, (2015).

[5] M. Bohner, S.G. Georgiev, Multivariable Dynamic Calculus on Time Scales, Springer Int. Publ. Switzerland, (2016) http://dx.doi.org/10.1007/978-3-319-47620-9.

[6] M. Bohner, A. Nosheen, J. Pecaric, A. Younas, Some dynamic Hardy type inequalities on time scales, J. Math. Inequal. 8 (1) (2014) 185-199.

[7] M. Bohner, A. Petereson, Dynamic Equations on Time Scales; An Introduction with Applications, Birkhauser, Boston, (2001).

[8] G.H. Hardy, Note on a theorem of Hilbert, Math. Z. 6 (3-4) (1920) 314-317, http://dx.doi.org/10. 1007/BF01199965.

[9] G.H. Hardy, Notes on some points in the integral caluclus, LX. An inequality between integrals, Messenger Math. 54 (1925) 150-156.

[10] G.H. Hardy, Notes on some points in the integral calculus, Messenger Math. 57 (1928) 12-16.

[11] G.H. Hardy, J.E. Littlewood, Elementary theorems concerning power series with positive coefficients and moment constants of positive functions, J. Reine Angew. Math. 157 (1927) 141-158.

[12] S. Hilger, Analysis on measure chains-a unified approach to continuous and discrete calculus, Results Maths (1990) 18-56.

[13] S.H. Saker, O'Regan Donal, Hardy and Littlewood Inequalities on time scales, Bull. Malays. Math. Sci. Soc. 39 (2) (2016) 527-543.

\section{Corresponding author}

Ammara Nosheen can be contacted at: hammaran@gmail.com
Inequalities of multivariate Hardy and Littlewood

For instructions on how to order reprints of this article, please visit our website:

www.emeraldgrouppublishing.com/licensing/reprints.htm

Or contact us for further details: permissions@emeraldinsight.com 